\title{
From natural dye to herbal medicine: a systematic review of chemical constituents, pharmacological effects and clinical applications of indigo naturalis
}

\author{
Yang Qi-yue ${ }^{1}$, Zhang Ting ${ }^{2}$, He Ya-nan ${ }^{2}$, Huang Sheng-jie ${ }^{2}$, Deng Xuan ${ }^{2}$, Han Li $^{2,3^{*}}$ and Xie Chun-guang ${ }^{1 *}$
}

\begin{abstract}
Background: Indigo naturalis is a blue dye in ancient, as well as an extensive used traditional Chinese medicine. It has a wide spectrum of pharmacological properties and can be used to treat numerous ailments such as leukemia, psoriasis, and ulcerative colitis. This article aims to expand our understanding of indigo naturalis in terms of its chemical constituents, pharmacological action and clinical applications.

Methods: We searched PubMed, web of science, CNKI, Google academic, Elsevier and other databases with the key words of "Indigo naturalis", and reviewed and sorted out the modern research of indigo naturalis based on our research results.

Results: We outlined the traditional manufacturing process, chemical composition and quality control of indigo naturalis, systematically reviewed traditional applictions, pharmacological activities and mechanism of indigo naturalis, and summarized its clinical trials about treatment of psoriasis, leukemia and ulcerative colitis.

Conclusions: Indigo naturalis has a variety of pharmacological activities, such as anti-inflammatory, antioxidant, antibacterial, antiviral, immunomodulatory and so on. It has very good clinical effect on psoriasis, leukemia and ulcerative colitis. However, it should be noted that long-term use of indigo naturalis may produce some reversible adverse reactions. In summarize, indigo naturalis is an extremely important drug with great value and potential.
\end{abstract}

Keywords: Indigo naturalis, Indole alkaloids, Anti-inflammation, Leukemia, Psoriasis, Ulcerative colitis

\section{Introduction}

Indigo naturalis is a dried powder or paste processed from the stems and leaves of Baphicacanthus cusia (Nees) Bremek. (family Acanthaceae), Polygonum tinctorium Ait. (family Polygonaceae) and Isatis indigotica Fort. Initially, indigo naturalis was used as a primitive dye in ancient India and then introduced into China through

\footnotetext{
*Correspondence: hanliyx@163.com;xcg718@yahoo.com.cn

${ }^{1}$ Hospital of Chengdu University of Traditional Chinese Medicine, No. 39 Shierqiao Road, Chengdu 610075, People's Republic of China

${ }^{3}$ Chengdu University of Traditional Chinese Medicine, No. 1188 Liutai Avenue, Chengdu 611137, China

Full list of author information is available at the end of the article
}

Persia [1]. In the Tang Dynasty,indigo naturalis is a fashionable cosmetic used for thrush. Subsequently, Chinese doctors found it could reduce fever, detoxify the blood, dissolve ecchymoses, and cool down blood [2]. Further study on the material basis of indigo naturalis showed that it was mainly composed of $10 \%$ organic matter and $90 \%$ inorganic substance, among which organic matter was the main active substance [3]. In addition, pharmacological effects of indigo naturalis were also studied. Modern researches found that indigo naturalis has antiinflammatory, antioxidant, antibacterial, immune regulation and other activities [4-6]. In recent years, indigo naturalis has also been found to be used in the treatment

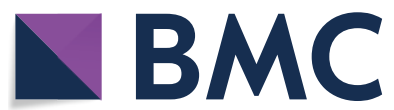

(c) The Author(s) 2020. This article is licensed under a Creative Commons Attribution 4.0 International License, which permits use, sharing, adaptation, distribution and reproduction in any medium or format, as long as you give appropriate credit to the original author(s) and the source, provide a link to the Creative Commons licence, and indicate if changes were made. The images or other third party material in this article are included in the article's Creative Commons licence, unless indicated otherwise in a credit line to the material. If material is not included in the article's Creative Commons licence and your intended use is not permitted by statutory regulation or exceeds the permitted use, you will need to obtain permission directly from the copyright holder. To view a copy of this licence, visit http://creativeco mmons.org/licenses/by/4.0/. The Creative Commons Public Domain Dedication waiver (http://creativecommons.org/publicdomain/ zero/1.0/) applies to the data made available in this article, unless otherwise stated in a credit line to the data. 
of cancer, especially indirubin and tryptanthrin have obvious inhibitory effects on various cancers $[7,8]$. The discovery of these bioactivities provided positive support for the clinical applications of indigo naturalis. In this manuscript, we reviewed the manufacturing process, chemical composition, traditional applications, pharmacological mechanisms and clinical applications of indigo naturalis, which can provide comprehensive and detailed references to related researchers and contribute to the further development and clinical applications of indigo naturalis.

\section{Manufacturing process}

The conventional manufacturing method includes collecting stems and leaves, soaking, producing coarse indigo, refining, and drying (Fig. 1). The manufacturing process is very interesting, including microbial action, component transformation, adsorption and precipitation (Fig. 2) [6]. Firstly, the stems and leaves from Baphicacanthus cusia (Nees) Bremek, Polygonum tinctorium Ait or Isatis indigotica Fort are soaked in water until rot. Under the action of microorganisms, indole glycosides and endogenous polyglucosidases are released from stems and leaves. In soaking processing, the most important chemical change is that endogenous polyglucosidases hydrolyzes indole glycoside to produce indole phenol, which is the source of indigo and indirubin. Then, stems and leaves are removed and lime slurry is added. Indole phenol combines with oxygen to form indole phenol free radical in an alkaline environment. Some indole phenol radicals condense to form indigo, and some indole phenol radicals are further oxidized to indole ketone and combine with indole phenol to form indirubin. Simultaneously, $\mathrm{Ca}(\mathrm{OH})_{2}$ in lime reacts with $\mathrm{CO}_{3}{ }^{2-}$ in the soaking solution to form the $\mathrm{CaCO}_{3}$ nucleus, which provides carriers for adsorbing indigo, indirubin and other compounds. With the continuous deposition of the compound on the surface of $\mathrm{CaCO}_{3}$, the gravity of the polymer exceeds the buoyancy, and the solid-liquid separation is realized at the bottom of the water. These polymers are the original indigo naturalis and commercial indigo naturalis is obtained from the further separation, purification and drying of the original indigo naturalis. In fact, the process of soaking and adding lime is not only the conversion of indole phenol, as well as the transformation of other substances. However, the transformation of other substances needs further researches.

\section{Chemical compositions}

Although inorganic substances in indigo naturalis occupy the majority, organic substances are main active components. The organic components can be divided into the following categories $[6,9,10]$. Besides, organic composition ratio of indigo naturalis may not be exactly the same, which depended on the source of stems and leaves.

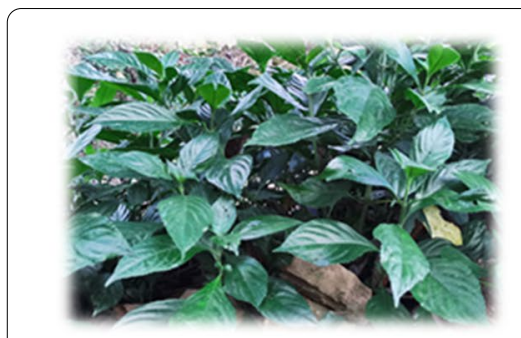

Stems and leaves of Isatis indigotica Fort, Polygomum tinctorium Ait or Baphicacanthus cusia (Nees) Bremek

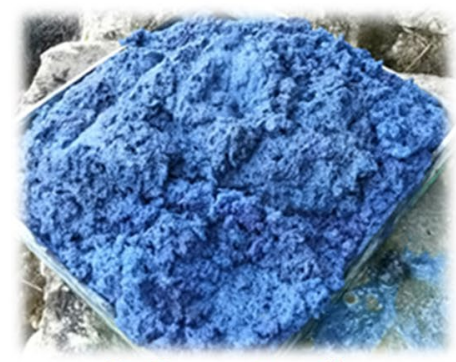

Indigo naturalis

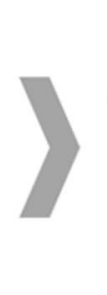

\section{.} .

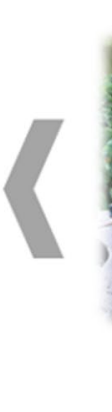

Constantly stirring Collecting sediment and flam

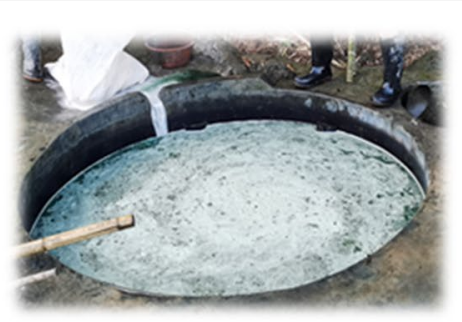

Removing stems and leaves Adding lime slurry

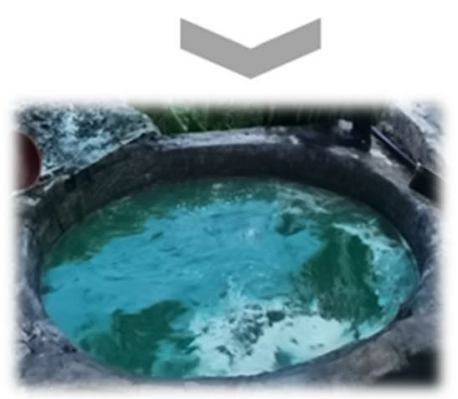

Adding lime slurry

Fig. 1 The manufacturing process of Indigo naturalis 


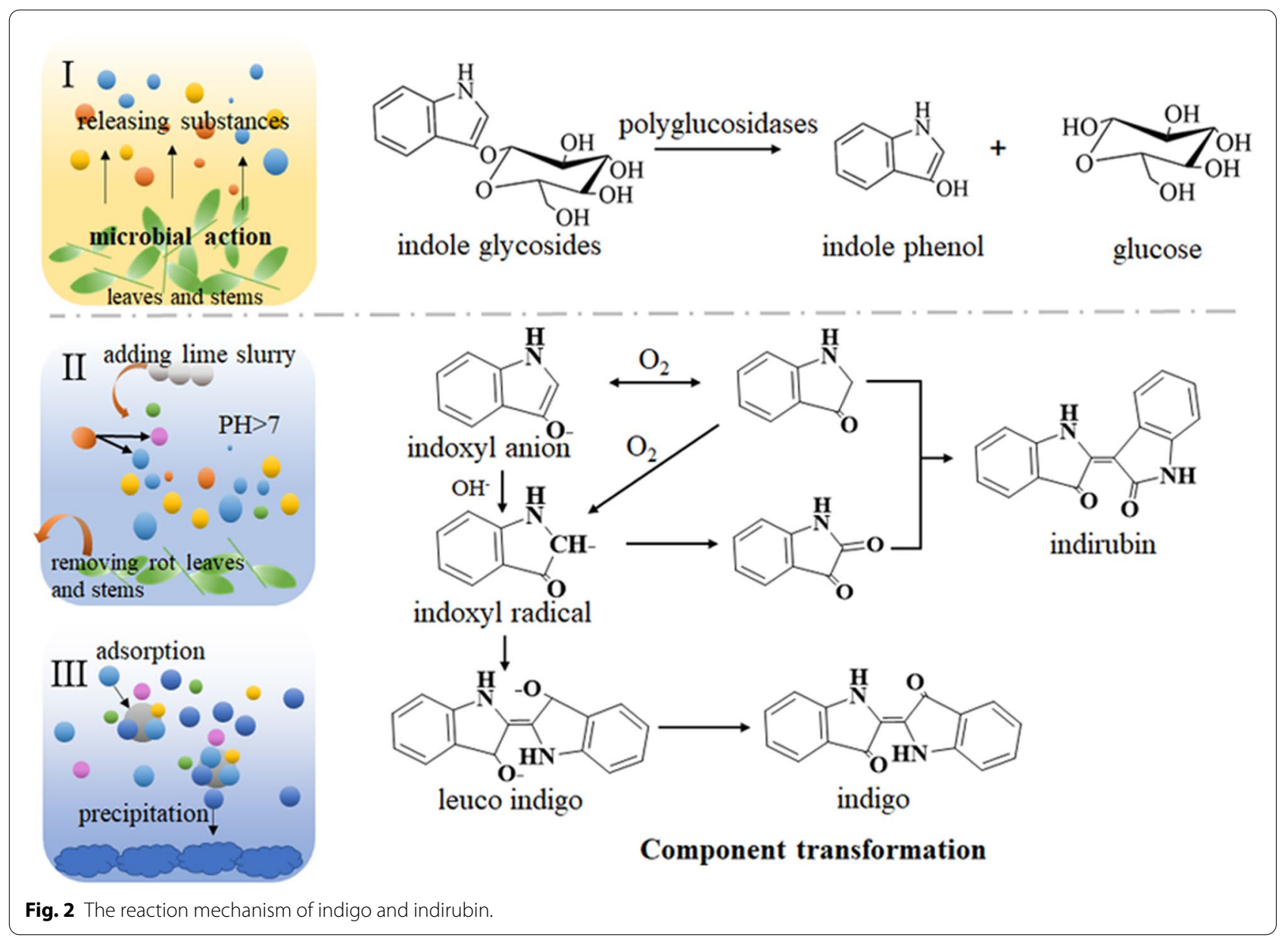

- Indole alkaloids include indigo (1), indirubin (2), isatin (3), isoindigo (4), hydroxyindirubin (5), 2-hydroxy-1,4-benzoxazin-3-one (6), tryptanthrine (7), quinazoline-2,4-dione (8), etc. (Fig. 3). Indigo, indirubin and isoindigo are two indole alkaloids; moreover, they are isomers.

- Nucleosides include uracil (9), cytidine (10), hypoxanthine (11), adenosine (12), adenine (13), inosine (14) and thymidine (15), etc.

- Sterol compounds include stigmasterol (16), taraxasterol (17), $\alpha$-sitosterol and $\beta$-sitosterol (18), etc.

- Amino acids include aspartate(19), threonine (20), serine (21), glutamic acid (22), proline (23), glycine (24), alanine (25), cystine (26), valine (27), methionine (28), leucine (29), isoleucine (30), tyrosine (31), phenylalanine (32), tryptophan (33), lysine (34), histidine (35), arginine (36), etc.

- Others include 2-aminobenzoic acid (37), N-phenyl-2-naphthylamine (38), lupenone (39), n-heptadecanoic acid (40), etc.
The inorganic ingredients of indigo naturalis was consist of $70 \%$ calcium carbonate, $10 \%$ silica dioxide and calcium hydroxide $[3,11]$. In addition, it may also include $\mathrm{Fe}_{2}\left(\mathrm{CO}_{3}\right)_{3}, \mathrm{Al}_{2}\left(\mathrm{CO}_{3}\right)_{3}$, etc [11]. Calcium carbonate and calcium hydroxide are derived from lime, and silica dioxide and other impurities are derived from soil.

\section{Quality control}

\section{Character evaluation}

Traditional quality evaluation parameters include shape, color, smell, texture, water to test, and fire to test. The best quality is fine powder, blue in color, light and loose in texture, with no sand and stone. In addition, it is able to float in the water, and the water is not blue. When it burns, it gives out a purplish red flame.

\section{Physical and chemical evaluation}

At present, thin layer chromatography (TLC) and highperformance liquid chromatography (HPLC) are the 
<smiles>O=C1/C(=C2\Nc3ccccc3C2=O)Nc2ccccc21</smiles>

(1) indigo<smiles>O=C1c2ccccc2-n2c1nc1ccccc1c2=O</smiles>

(7) tryptanthrine

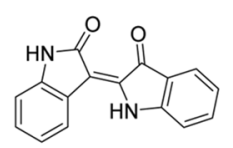

(2) indirubin<smiles>O=c1[nH]c(=O)c2ccccc2[nH]1</smiles>

(8) quinazoline2,4-dione

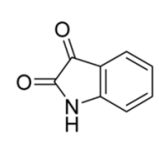

(3) isatin<smiles>O=c1cc[nH]c(=O)[nH]1</smiles>

(9) uracil

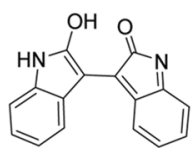

(4) isoindigo

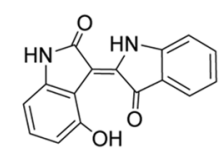

(5) hydroxyindirubin<smiles>O=C1Nc2ccccc2OC1O</smiles>

(6) 2-hydroxy-1,4benzoxazin-3-one<smiles>Cc1cn([C@H]2C[C@H](O)[C@@H](CO)O2)c(=O)[nH]c1=O</smiles><smiles>Nc1ccn(C2OC(CO)C(O)C2O)c(=O)n1</smiles><smiles>O=c1[nH]cnc2[nH]cnc12</smiles><smiles>Nc1ncnc2c1ncn2[C@@H]1O[C@H](CO)[C@@H](O)[C@H]1O</smiles>

(10) cytidine

(11) hypoxanthine

(12) adenosine

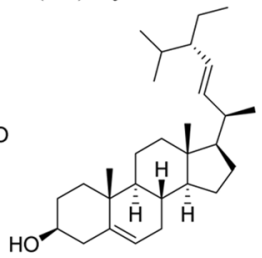

(16) stigmasterol

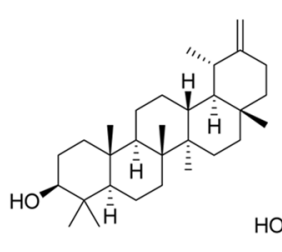

(17) taraxasterol

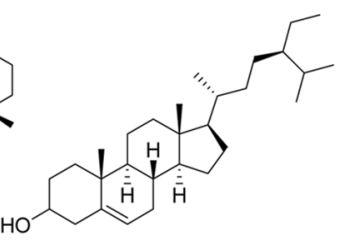

(18) $\beta$-sitosterol

(13) adenine

(14) inosine

(15) thymidine<smiles>N[C@@H](CCC(=O)O)C(=O)O</smiles><smiles>O=C(O)C1CCCN1</smiles><smiles>NCC(=O)O</smiles><smiles>N[C@@H](CC(=O)O)C(=O)O</smiles><smiles>CC(O)C(N)C(=O)O</smiles><smiles>N[C@@H](CO)C(=O)O</smiles>

(20) threonine

(21)serine

(22) glutamic acid

(23) proline

(24) glycine<smiles>CC(N)C(=O)O</smiles><smiles>N[C@@H](CCSSC[C@H](N)C(=O)O)C(=O)O</smiles><smiles>CC(C)[C@H](N)C(=O)O</smiles><smiles>CSCCC(N)C(=O)O</smiles>

(28) methionine

(29) leucine<smiles>CC(C)(C)C(N)C(=O)O</smiles><smiles>CCC(C)C(N)C(=O)O</smiles>

(25) alanine

(26) cystine

(27) valine<smiles>NC(Cc1c[nH]c2ccccc12)C(=O)O</smiles><smiles>NCCCC[C@H](N)C(=O)O</smiles><smiles>NC(Cc1c[nH]cn1)C(=O)O</smiles><smiles>N=C(N)NCCCC(N)C(=O)O</smiles>

(31) tyrosine

(32) phenylalanine

(33) tryptophan

(34) lysine

(35) histidine

(36) arginine

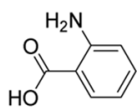

(37) 2-aminobenzoic acid<smiles>c1ccc(Nc2ccc3ccccc3c2)cc1</smiles>

(38) N-phenyl-2naphthylamine

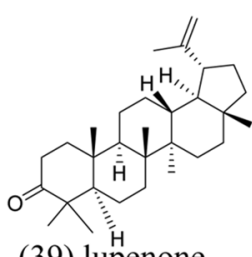

(39) lupenone

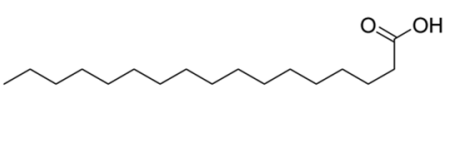

(40) n-heptadecanoic acid

Fig. 3 Structural formula of some chemical constituents in indigo naturalis

most commonly used quality control methods for indigo naturalis. For instance, the 2015 Chinese pharmacopoeia used TLC to identify indigo and indirubin in samples. The indigo content was more than $2 \%$ and that of indirubin was more than $0.13 \%$. Besides TLC and HPLC, there are many other quality control methods for indigo naturalis. Degani et al. [11] describe an analytical procedure based on the silylating agent N,O-bis-(trimethylsilyl) trifluoroacetamide with $1 \%$ trimethylchlorosilane for pure molecules of indigo, indirubin and plant extracts of indigo naturalis. Gas Chromatography-mass spectrometry turns out to be an efficient and fast analytical 
tool for the identification of natural indigo in plants and textile artifacts. Li et al. [9] established a reversed phase HPLC method of simultaneous determination of five nucleosides in indigo naturalis: uracil, cytidine, hypoxanthine, inosine, and thymidine. The method was applied to compare the contents of nucleosides in indigo naturalis from different producing areas and batches. It was found that there were obvious differences in nucleoside content of indigo naturalis from different producing areas or batches. Liao et al. [12] determined the contents of copper, arsenic, cadmium, lead, and mercury in indigo naturalis by inductively coupled plasma mass spectrometry. This method can accurately and rapidly determine the content of heavy metals mentioned above. It is very useful for controlling the quality of indigo naturalis and ensuring its safety.

\section{Biological evaluation}

Indigo naturalis has clear antiviral activity, as dose Radix Isatidis [13]. Neuraminidase (NA) is a necessary enzyme for replication of the influenza virus. At present, blocking the function of neuraminidase is recognized as an effective way to treat influenza presently. Oseltamivir and zanamivir are commonly used neuraminidase inhibitors, which commonly used to prevent and treat influenza. Therefore, our research team developed a biological assay to characterize the antiviral activity of indigo naturalis by determining the inhibitory rate of neuraminidase activity $[14,15]$. The neuraminidase-based bioassay was highthroughput and simple, and could be used for evaluation and quality control of indigo naturalis.

Different from other traditional Chinese medicine, indigo naturalis has the characteristics of diverse plant sources and complex manufacturing technology. The quality of indigo naturalis can be affected by plant source, ratio of plant raw materials to lime, soaking time, climate and production season, which makes it difficult to standardize and standardize the manufacturing of indigo naturalis. At present, researches on the quality control of indigo naturalis are mainly focused on the organic compounds such as indigo, indirubin and tryptamine. However, lime is an essential raw material in the process of indigo naturalis manufacture. There are few studies on the problem of adding excessive lime. Shi et al. [16] found that many commercial indigo naturalis is a qualified product according to the standard, but most of them have high the total ash content and strong alkaline due to the addition of excessive lime. Obviously, excessive lime will affect the quality of indigo naturalis, and even affect the clinical efficacy and safety. Therefore, researchers should also focus on the control of inorganic content in indigo naturalis, except for the control of organic matter content.

\section{Traditional applications}

It is easy to see from the name of indigo naturalis that this plant originated in India. The earliest literature records that indigo naturalis is abundant in Caoguo (now located in Afghanistan) and Turan (now located in Kazakhstan). Persians use indigo to dye their hair, and Chinese women use it to paint their eyebrows. In Natural History, Pliny mentions that indigo naturalis from the East was used by ancient Romans as paint, applied around the eyes as a cosmetic, or used as medicine, to promote wound healing. Since the Tang Dynasty, indigo naturalis has been used as a drug with remarkable therapeutic effects on fever, hemoptysis, convulsions in children, oral ulcers and sore throat. The earliest medical records in China appeared in the Theory of Medicinal Properties (Tang Dynasty, 627 A.D.). Later, it was included in many other famous medicinal books, including Kaibao Materia Medica (Song Dynasty, 973 A.D.), Materia Medica Yanyi (Song Dynasty, 1116 A.D.), and Compendium of Materia Medica (Ming Dynasty, 1590 A.D.). Indigo naturalis is indicated for various kinds of bleeding syndromes, such as hemoptysis and epistaxis, and its powder can be dipped with sterilized cotton and stuffed into the nasal cavity to stop bleeding. Indigo naturalis also can be used to cure oral diseases, such as a swollen, sore throat, ulcers, swelling, pain, and rottenness of mouth and tongue. In Supplements to Commentarieson the Synopsis of the Golden Chamber, it is used in a powder that is blown into the infected region. In addition to its use as a topical drug, indigo naturalis has been used in the past to treat convulsions and epilepsy in children. To increase its clinical effect, indigo naturalis is usually used in combination with other Chinese herbs, such as Gypsum Fibrosum, Bezoar, Snakegourd Seed and Radix Bupleuri.

\section{Pharmacology and mechanisms Antioxidation}

Excessive oxygen free radicals can cause aging, inflammation and other diseases, which affect human health. Some components of medicinal plants have natural antioxidant effect, which is important in preventing and treating diseases caused by abnormal increase of oxygen free radicals [17]. It has been shown that indigo naturalis can play an antioxidant role by scavenging oxygen free radicals, improving the activity of superoxide dismutase, inhibiting the production of malondialdehyde, and stabilizing cell membrane permeability [18]. Lin [19] found that the extract of indigo naturalis could inhibit the increase of reactive oxygen species (ROS), $\mathrm{H}_{2} \mathrm{O}_{2}$ or derived hydroxyl radicals induced by exogenous $\mathrm{H}_{2} \mathrm{O}_{2}$. Meanwhile, it could interfere with protein modification induced by a lipid peroxidation product 4-hydroxy-2-nonenal (HNE), and protect keratinocytes from the electrophilic attack 
of HNE. Zhao [20] found that indigo and indirubin, the main components of indigo naturalis, could effectively scavenge superoxide anion. The mechanism may be associated with some electrophilic groups, such as ketones or aldehydes. These electrophilic groups could promote the release of hydrogen ion from hydroxyl groups and form non free radical superoxide anion, thus stabilizing superoxide anion. Another study found that indigo and indirubin could promote clearing of ROS, reduce the malondialdehyde content, promote the balance between the antioxidant system and ROS, inhibit lipid peroxidation, and protect cell membranes from oxidative damage [21]. Indigo can protect gastric mucosa from ethanolinduced gastric injury by preventing ethanol-induced DNA damage through non-enzymatic antioxidation and inhibition of polynuclear neutrophil infiltration [22]. What's more, indigo naturalis can reduce the release of proinflammatory factors TNF- $\alpha$ and IL-6, indirectly reduce the level of ROS, and play the role of oxygen oxidation [23]. These results indicate that indigo naturalis and its some main components have potential as antioxidants.

\section{Anti-inflammatory effects}

Inflammation, characterized by redness, swelling, heat and pain, is a body's defense response to stimulation. However, excessive or long-term inflammatory response can lead to tissue damage [24]. Indigo naturalis and its some active ingredients have been proved to have significant anti-inflammatory effects, whose mechanism is the down-regulation of some inflammation factors and the inhibition of NO production $[25,26]$. For instance, indigo naturalis and its main compound indigo can reduce the expression of TNF- $\alpha$, IL-1, IL-6, IL-10, IL-22 and other inflammatory factors in plasma of $\mathrm{UC}$ mice via antagonizing TLRs/NF- $\kappa$ B pathway and activating AhR pathway, thus improving inflammation [27, 28]. Indigo naturalis also can improve the oxidative stress level of colon tissue by increasing superoxide dismutase (SOD) and decreasing malondialdehyde (MDA) level, so as to control and reduce inflammatory reaction [29]. Indirubin inhibits the production of inflammatory cytokines through a variety of signaling pathways, and has a certain dose-dependent $[30,31]$. A study found that indirubin significantly inhibited TLR4 and NF- $\kappa$ B signaling and decreased the level of inflammatory cytokines in mice with lipopolysaccharide (LPS)-induced acute lung injury [25]. Except for the NF- $\kappa$ B signaling pathway, indirubin also inhibits the MAPK signaling by decreasing the phosphorylation of extracellular ERK, P38, and JNK in LPS-induced mouse mastitis [32]. Another study found that indirubin can also reduce crypt deformation and mucosal damage, and reduce the infiltration of inflammatory cell in colonic mucosa [33]. Additionally, indigo naturalis can also play an anti-inflammatory role by inhibiting intracellular $\mathrm{Ca}^{2+}$ concentration. In formyl-L-methionyl-L-leucyl-L-phenylalanine (FMLP)-activated human neutrophils, indigo naturalis could significantly reduce $\mathrm{O} 2(.-)$ generation and elastase release, inhibit calcium mobilization and reduce phosphorylation of extracellular p38 MAPK, JNK and some regulated kinase [34]. While indigo naturalis not affect cellular cAMP levels. Interestingly, neither indirubin, indigo nor tryptanthrin had similar effects in FMLPactivated human neutrophils and this result indicates that there are some unknown activities. Although indigo naturalis has significant anti-inflammatory effect, its antiinflammatory mechanism and active ingredients need to be further explored.

\section{Antibacterial}

Indigo naturalis and its active components have good antibacterial, antifungal and antiviral activities [35-37]. The antimicrobial assays in vitro suggested that the ethyl acetate extract of indigo naturalis could remarkably inhibit gram-positive bacteria (methicillin-resistant Staphylococcus aureus, Staphylococcus epidermidis, and Staphylococcus aureus) and mildly inhibit noncutaneous fungal pathogens of onychomycosis (Aspergillus fumigatus and Candida albicans) [38]. For these bacteria, tryptanthrin has better antibacterial activity than indigo. It could suppress the growth of pathogens by intercalating into bacterial DNA [39]. Indigo can cause extensive oxidative damage so as to host cell death by promoting superoxide production [40]. In the absence of light, indigo can have antibacterial effects on Staphylococcus aureus, Staphylococcus epidermidis, Escherichia coli, Proteus vulgaris, and Candida albicans (minimum inhibitory concentration $[\mathrm{MIC}]=240 \mu \mathrm{m}$ ). Interestingly, in the presence of light, a lower dose of indigo $(\mathrm{MIC} / 2)$ can show antibacterial effects [40]. Indirubin can also inhibit Staphylococcus aureus and enhance the activity of ciprofloxacin. This mechanism may be interrelated to the inhibition of NorA efflux pump in Staphylococcus aureus [41]. Indigo is the isomer of indirubin, but there is no related research on its inhibition of NorA efflux pump.

\section{Antiviral}

Indole alkaloids, especially bisindole nuclear alkaloids, have been widely concerned in the field of anti-infection due to their special structure, strong analogues, high selectivity and low side effects [42]. Indigo naturalis is rich in indole alkaloids, and the anti-virus research of indigo naturalis mainly focuses on indigo and indirubin. Influenza virus is the pathogen of influenza, especially 
influenza A virus is easy to mutate, causing a worldwide pandemic [43]. Indirubin could reduce activation of the chemokine regulated on, the expression of normal $\mathrm{T}$ cell and the secretion of influenza virus A/NWS/33- infected H292 human epithelial cell line. And this inhibitory effect was also observed as well as influenza virus B/ Lee-infected cells [44]. In vivo test, Indirubin can reduce the sensitivity of stress-induced mice to influenza virus (H1N1), thereby reducing lung injury and improving survival rate [36]. Further study showed that indirubin could promote the formation of IFN-glycolic acid and increase the expression of IFN- $\beta$ and IFN-induced transmembrane 3 through mitochondrial antiviral signal copper aluminum, thus maintaining the morphology and function of mitochondria after $\mathrm{H} 1 \mathrm{~N} 1$ infection. Indigo and indirubin have strong killing activity against Japanese encephalitis virus, which may be related to block virus attachment. Especially indirubin has a strong protective effect on lethal Japanese encephalitis virus model [45]. In addition, indigo can inhibit the $3 \mathrm{C}$-like protease $\left(3 \mathrm{CL}^{\mathrm{pro}}\right)$ of SARS-coronavirus in dose dependence, which can induce the hydrolysis of replication enzyme polypeptides $1 \mathrm{a}$ and $1 \mathrm{ab}$ into functional proteins [45]. The above studies indicate that indigo naturalis and its active ingredients are expected to become potential antiviral drugs, especially drug-resistant viruses. Meanwhile, more in-depth experiments are needed to prove their potential as antiviral drugs.

\section{Immune modulation}

Indigo naturalis, as traditional Chinese medicine, has been widely used in autoimmune diseases and inflammatory diseases, such as psoriasis and allergic contact dermatitis $[46,47]$. A study has shown that indigo naturalis can inhibit oxazolidone induced allergic dermatitis by reducing eosinophil recruitment and production of Th2 related cytokines. But at the same time, indigo naturalis can change the structure of intestinal flora and aggravate colitis [48]. Indirubin can decrease serum IgE and cytokines production, and normalize the expression of NF- $\kappa \mathrm{B}, \mathrm{I} \kappa \mathrm{B}-\alpha$ and MAP kinase expression in a dose dependence by regulating the co-expression of Th1 and Th2-mediated immune response [47]. Indirubin can also improve the proliferation of keratinocytes, inhibit the activation of JAK3/STAT3 pathway, reduce the osmosis of CD $3+\mathrm{T}$ cells, $\gamma \delta \mathrm{T}$ cells and CD11b + neutrophils, and decrease the level of $\gamma \delta \mathrm{T}$ cells in spleen and lymph nodes [49]. What's more, indirubin can selectively increase the number of CD4 + CD25 + Treg cells, which is important in the induction and maintenance of peripheral selftolerance, leading to the induction of immune tolerance [50]. Man et al [51]. detected the effect of indirubin on ATP-induced macrophage immune response: neutral red dye uptake was used to measure cell death, and the ROS level was tested by dihydroethidium fluorescence probe. The results showed that indirubin could reverse the ATP-induced decrease in phagocytosis and cell death, and inhibit ATP-induced ROS production, indicating that indirubin blocked ATP-induced macrophage immune response.

\section{Antitumor}

Malignant tumor has become one of the major diseases threatening human beings. It is urgent to find drugs with high selectivity, small side effects and strong curative effect. Among numerous pharmacological actions of indigo naturalis, the antitumor effect is of great interest. Indirubin is of importance in the antitumor effects of indigo naturalis. In recent years, indirubin in indigo naturalis has attracted more and more attention in the field of anti-tumor. It has significant antiproliferative activity and is a powerful apoptosis inducer for a variety of tumor cells, such as cervical cancer, liver cancer and lymphoma cell lines [52, 53]. Realgar-indigo naturalis, a traditional Chinese medicine preparation composed of indigo naturalis, Salvia miltiorrhiza and realgar, was used to treat human acute promyelocytic leukemia (APL). The complete remission rate of APL patients with Realgar-indigo naturalis was 96.7-98\%, and the 5-year overall survival rate was $86.88 \%[54,55]$. Wang et al. [56] observed the effects of tetrasulfide (A) in realgar, indirubin (I) in indigo naturalis and tanshinone IIA (T) in Salvia miltiorrhiza in vivo and in vitro. They found that compared with single component or dual drug combination, ATI can increase the ubiquitination/degradation of promyelocytic leukemia (PML)-retinoic acid receptor $\alpha$ (RAR $\alpha$ ) oncoprotein, enhance the reprogramming of myeloid differentiation regulators, and enhance $G_{1} / G_{0}$ block in APL cells by striking multiple targets. In addition, ATI enhanced the level of Aquaglycoporin 9, promoted the transport of a to APL cells, and then promoted the degradation and therapeutic effect of PML-RAR $\alpha$ mediated by A. In the cell viability and induce apoptosis test of 2 human ovarian cancer cell lines, indirubin can inhibit cell activity and induce apoptosis with dose dependence by downregulating the phosphorylation of STAT3 (tyr705), and significantly suppressing the expression of cyclin D1 and c-myc downstream of STAT3 [31]. Indirubin can inhibit tumor necrosis factor induced NF- $\kappa \mathrm{B}$ activation in a dose and time-dependence. It can also inhibit the expression of NF $-\kappa \mathrm{B}$ regulated genes, including anti apoptotic genes (iap1, iap2, Bcl-2, BCL XL and TRAF1), proliferation genes (cyclin D1 and c-myc), and invasive genes (COX-2 and MMP-9), thus playing anti-inflammatory and antitumor activities [30]. Indirubin can not only inhibit the proliferation of cancer cells, but also the angiogenesis of 
tumor growth process. A study found that indirubin suppressed JAK/STAT3 signaling pathway to inhibit tumor angiogenesis [57] .

Tryptanthrin in indigo naturalis also has antitumor activity. Tryptanthrin can make the murine myeloid leukemia WEHI-3B JCS cells stay in $\mathrm{G}_{0} / \mathrm{G}_{1}$ phase, and downregulate the expression of cyclin $\mathrm{D} 2, \mathrm{D} 3, \mathrm{CDK} 2,4,6$ genes, so as to inhibit the proliferation of WEHI-3B JCS and play an anti-tumor role [58]. In non-melanoma skin cancer, tryptanthrin can inhibit the proliferation of hair follicle cells by inhibiting the activation of ERK $\mathrm{E}_{1 / 2}$ and $\mathrm{p} 38$ and reducing the expression of $\beta$-Catenin [59]. Tryptanthrin can also inhibit angiogenesis by regulating VEGFR2 and $E K_{1 / 2}$ signaling pathways and reducing the level of Ang1, PDGFB and MMP2 [60]. Additionally, tryptanthrin can be used as a potential chemotherapy adjuvant to inverse multidrug resistance in a breast cancer cell line MCF-7 by down-regulating multidrug resistance gene 1 [61].

\section{Others}

Indirubin in indigo naturalis can inhibit wnt $/ \beta$-Catenin signal transduction by restoring the expression of wif1 , inhibit the expression of TGase 1 and down-regulate CDK1 to inhibit the proliferation of keratinocytes [6264]. Tryptophan, another active component of indigo naturalis, has been proved to have the ability to inhibit keratinocytes, which is stronger than indigo and indirubin $[65,66]$. Tryptophan can also inhibit the activity of apelin promoter, and inhibit the proliferation and migration of vascular endothelial cells by causing $\mathrm{G}_{2} / \mathrm{M}$ phase arrest, inhibiting Akt and FAK pathway, thus achieving anti angiogenesis effect [67]. Other studies found that indigo naturalis could repair intestinal mucosa. It can increase the expression of vascular endothelial growth factor, epidermal growth factor, and occludin protein, and promote the repair of intestinal mucosa [68]. Except for promoting intestinal mucosal repairing and ulcer healing, indigo naturalis also can inhibit the gene expression of MMP-1 and TIMP-1 and reduce the damage of colonic mucosa by inhibiting Smads signaling pathway and activating MAPK signal pathway [69, 70]. Intestinal microflora is a hot spot in the treatment of ulcerative colitis in recent years. Indigo naturalis was found that it could reduce the relative abundance of harmful bacteria in the colon, increase the relative abundance of probiotics, and maintain the intestinal microbial homeostasis [71].

\section{Clinical applications}

\section{Acute promyelocytic leukemia}

APL is a common malignant disease in the blood system and characterized by abnormal coagulation function and acute promyelocytosis [72]. All trans retinoic acid (ATRA) and arsenic therapies are the most widely used therapies for APL, which has serious adverse reactions, high toxicity and relapse rate [73]. Indigo naturalis alone has no effect on APL, but indirubin in indigo naturalis could enhance the inhibition of arsenic disulfide in realgar on the proliferation and apoptosis of diffuse large celllymphoma cells and promote the transport of arsenic disulfide to APL cells, thus enhancing the degradation and treatment effect of PML-RAR $\alpha$ mediated by arsenic disulfide and accelerating the apoptosis of NB4 cells $[56,74]$. Clinical studies showed that the relapse rate of APL was $12.90 \%$ and the total mortality rate was $6.45 \%$ by alternately using chemotherapy and realgar-indigo naturalis formula (RIF), mainly consist in realgar and indigo naturalis. Compared with ATRA therapy, there was no significant difference in blood parameters $[8,75]$. It should be noted that patients with RIF may have adverse reactions such as nausea, vomiting, diarrhea, abdominal pain and rash, but there are no serious adverse reactions [76]. In non-high-risk APL clinical trial, the results showed that oral RIF plus ATRA has the same efficacy as intravenous arsenic trioxide plus ATRA and can replace the latter [77]. The adverse reactions of the two therapies were compared. It was found that liver aspartate aminotransferase or alanine aminotransferase concentration increased in 9\% patients with RIF plus ATRA and 6\% patients with arsenic trioxide plus ATRA, and grade 2-3 infection occurred in 14\% patients with RIF plus ATRA and $23 \%$ patients with arsenic trioxide plus ATRA. In the arsenic trioxide-ATRA group, there are two patients died during induction therapy. In children with APL, the 5 -year event-free survival of RIF group is $100 \%$, as well as intravenous arsenic trioxide after an average follow-up of 3 years. The adverse reactions were mild, but the hospitalization time of RIF was significantly lower than that of intravenous arsenic trioxide [78]. The above studies show that indigo naturalis is a potential adjuvant drug for treating APL.

\section{Psoriasis}

Psoriasis is a chronic immune related skin disease. It is characterized by erythema, scales and pruritus, which is determined by polygenic inheritance and induced by multiple environmental factors [79]. The clinical observation of indigo naturalis in treating psoriasis has been reported in the $1980 \mathrm{~s}$, and the overall cure rate is $69.6 \%$ [80]. According to the modern research results, indigo naturalis mainly treats psoriasis by inhibiting the excessive proliferation of keratinocytes, regulating the body immunity, reducing inflammation and anti angiogenes [62-65, 67]. At present, indigo naturalis is mostly used as external preparation in treating psoriasis vulgaris and nail psoriasis (Table 1). It should be noted that in a clinical report, 4 out of 42 subjects had pruritus after using 


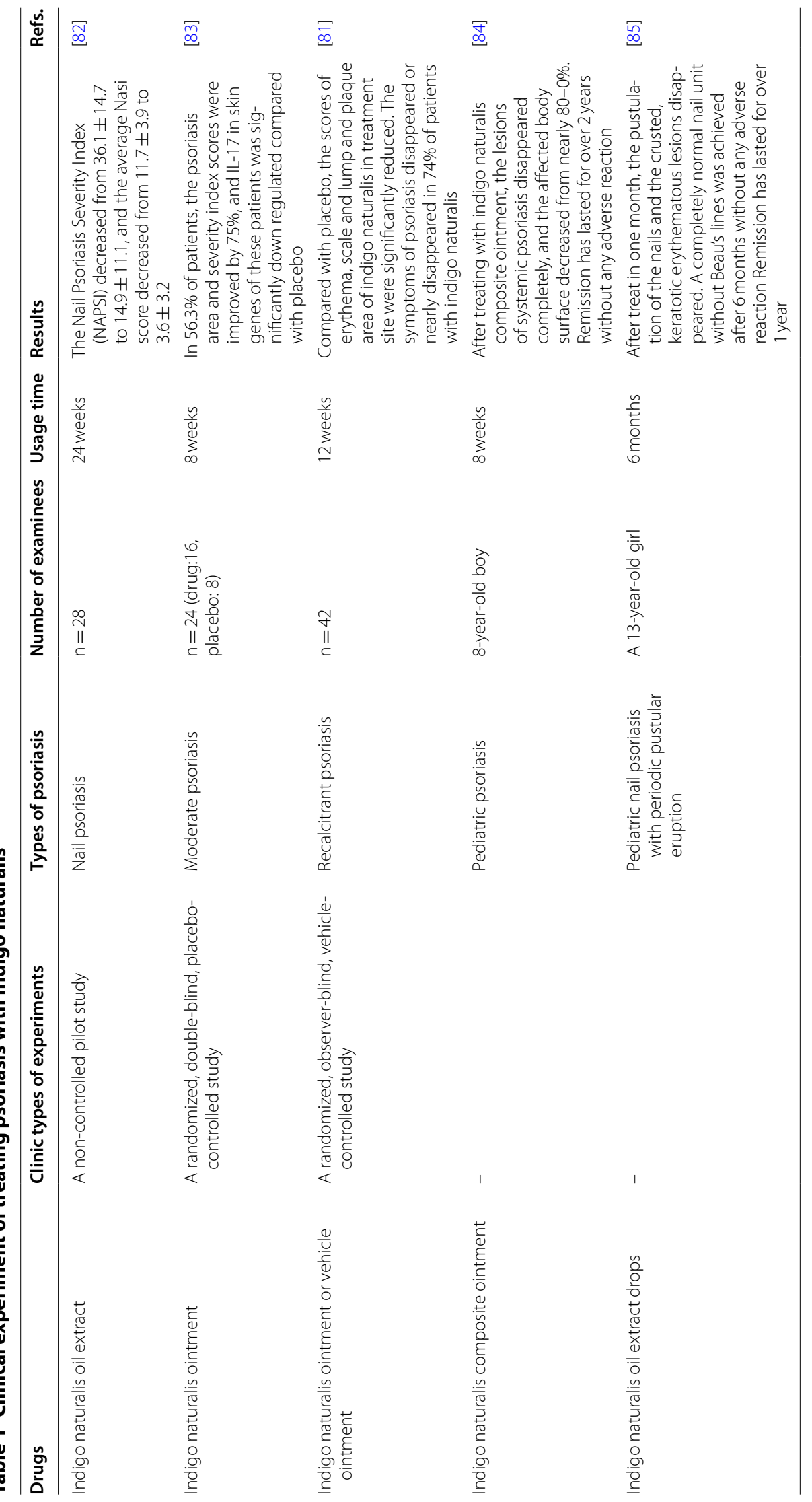




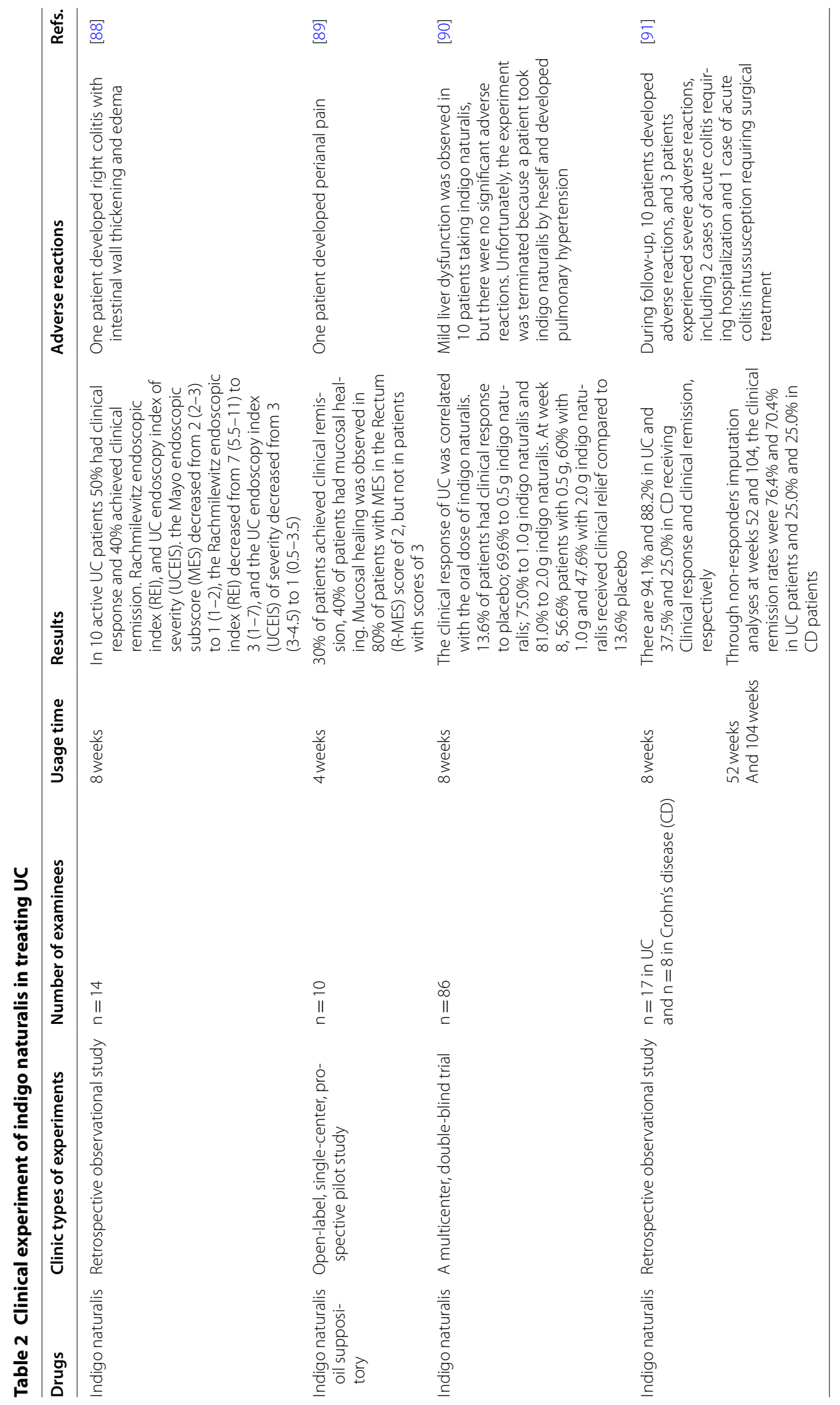


indigo naturalis ointment [81]. The safety of indigo naturalis for external use needs further evaluation. Moreover, the indigo naturalis ointment are prepared by the hospital itself without no unified standard. Therefore, it is necessary to develop indigo naturalis into qualified patent medicine as soon as possible.

\section{Ulcerative colitis (UC)}

$\mathrm{UC}$ is a common clinical digestive tract disease, mainly manifested as abdominal pain, diarrhea and bloody stool, and intestinal mucosal ulcer, erosion, bleeding and so on can be seen under the microscope [86]. According to relevant reports, indigo naturalis mainly treats ulcerative colitis by reducing inflammation, repairing intestinal mucosa and regulating intestinal flora [33, 69-71]. According to clinical observation results of indigo naturalis, it has curative effect in the treatment of UC. At the same time, there are also adverse reactions such as gastrointestinal reactions, liver injury and pulmonary hypertension, but there is no death (Table 2). In order to further research the safety of indigo naturalis in treating UC, a follow-up survey was carried out in 877 patients using indigo naturalis. It was found that 91 patients (107 cases) had adverse reactions, including 40 cases of liver dysfunction, 21 cases of gastrointestinal symptoms, 13 cases of headache and 11 cases of pulmonary hypertension. Liver dysfunction is mildly reversible. Surgery is required in $40 \%$ of patients with intestinal adhesions. After stopping the treatment, pulmonary hypertension could heal itself without any death [87]. Although indigo naturalis has some side effects in the treatment of UC, it is still a potential drug. Meanwhile, it is necessary to monitor adverse reactions of indigo naturalis for a longtime use.

\section{Other clinical applications}

In addition to the treatment of leukemia, psoriasis and colitis, modern research has found that indigo naturalis can also be used to treat liver injury, nephritis, mouth ulcer and other skin diseases, which may be related to its anti-inflammatory, antioxidant and immune regulatory effects [18, 20, 92, 93]. However, research of these diseases mostly stays in the basic stage, and more data support is needed from the clinical use.

\section{Application challenges and solutions}

In recent years, indigo naturalis has been widely used in the treatment of leukemia, psoriasis, ulcerative colitis and other diseases, as well as been recognized by experimental research and clinical observation. However, it seems that indigo naturalis lacks accurate dose-response relationship research and direct clinical big data to guide clinical application, especially its safety. Therefore, it is necessary to carry out clinical observations with randomized controlled, double-blind, double-dummy, multi-center and large sample, which is conducive to the further development and clinical application of indigo naturalis. Additionally, it should speed up the development of indigo naturalis preparation to be convenient for the clinical application and improve the safety of indigo naturalis.

\section{Conclusion}

As a national medicine with a long history, indigo naturalis has a variety of biological activities and broad application prospects. Although the anti-inflammatory and anti-tumor effects of indigo naturalis have been widely recognized by the medical community, there are some adverse reactions such as gastrointestinal reactions, skin itching, reversible liver dysfunction and so on, which can not be ignored. The advantages and disadvantages should be fully considered in the use of indigo naturalis. Additionally, researches of indigo naturalis mainly focused on indigo, indirubin and tryptophan, but less on other components. It is necessary to use modern pharmaceutical research methods to study the efficacy and mechanism of its effective components. Meanwhile, it can also be used for drug design of indigo naturalis and its active ingredients to reduce adverse reactions, which makes indigo naturalis safer in clinical application.

\section{Abbreviations}

ROS: Reactive oxygen species; HNE: Lipid peroxidation product 4-hydroxy2-nonenal; LPS: Lipopolysaccharide; FMLP: Formyl-L-methionyl-L-leucyl-L-phenylalanine; APL: Acute promyelocytic leukemia; PML: Promyelocytic leukemia; RARa: Retinoic acid receptora; ATRA: All trans retinoic acid; RIF: Realgar-Indigo naturalis formula; UC: Ulcerative colitis.

\section{Acknowledgements \\ We thank Dr. Zhang Chen and Dr. Zhang Ding-kun for providing suggestions on this manuscript.}

\section{Authors' contributions}

YQY, XCG, and HL designed the study; ZT, DX, HYN and HSJ collected and analyzed the data; YQY, ZT, and HYN wrote the paper. All authors read and approved the final manuscript.

\section{Funding}

We are grateful to the support of National Natural Science Foundation Project (81773918)

\section{Availability of data and materials}

The datasets used in this study are available from the corresponding author upon reasonable request.

Ethics approval and consent to participate Not applicable.

\section{Consent for publication \\ The manuscript is approved by all authors for publication.}

Competing interests

The authors declare no competing financial interests. 


\section{Author details}

${ }^{1}$ Hospital of Chengdu University of Traditional Chinese Medicine, No. 39 Shierqiao Road, Chengdu 610075, People's Republic of China. ${ }^{2}$ School of Pharmacy, Chengdu University of Traditional Chinese Medicine, Chengdu, People's Republic of China. ${ }^{3}$ Chengdu University of Traditional Chinese Medicine, No. 1188 Liutai Avenue, Chengdu 611137, China.

Received: 28 October 2020 Accepted: 26 November 2020 Published online: 14 December 2020

\section{References}

1. Teng J. Textual research on indigo naturalis and the medicinal blue herbs. Chin Herbal Med. 1996;27:110-2.

2. Sun $X \mathrm{~L}, \mathrm{Lv}$ GH, Zhu CH, et al. Research on Application of Qingdai (Natural Indigo) in History. Acta Chin Med. 2020;35:1653-5.

3. Pan M, Pei W, Yao Y, et al. Rapid and Integrated Quality Assessment of Organic-Inorganic Composite Herbs by FTIR Spectroscopy-Global Chemical Fingerprints Identification and Multiple Marker Components Quantification of Indigo Naturalis (Qing Dai). Molecules. 2018;23:2743.

4. Gaitanis G, Magiatis P, Velegraki A, et al. A traditional Chinese remedy points to a natural skin habitat: indirubin (indigo naturalis) for psoriasis and the Malassezia metabolome. Br J Dermatol. 2018:179:800.

5. Naganuma M. Treatment with indigo naturalis for inflammatory bowel disease and other immune diseases. Immunol Med. 2019:42:16-21.

6. Yu H, Li TN, Ran Q, et al. Strobilanthes cusia (Nees) Kuntze, a multifunctional traditional Chinese medicinal plant, and its herbal medicines: A comprehensive review. J Ethnopharmacol. 2020;265:113325.

7. Li Y, Ligr M, McCarron JP, et al. Natura-alpha targets forkhead box $\mathrm{m} 1$ and inhibits androgen-dependent and -independent prostate cancer growth and invasion. Clin Cancer Res. 2011;17:4414-24.

8. Adès L, Guerci A, Raffoux E, et al. Very long-term outcome of acute promyelocytic leukemia after treatment with all-trans retinoic acid and chemotherapy: the European APL Group experience. Blood. 2010;115:1690-6

9. Li YS, He XR, Yang YY, et al. RP-HPLC Method for the Determination of Nucleosides in Indigo naturalis. Chin Med J Res Prac. 2018;32:56-8.

10. Wu YB, Wu JG, Wag HM, et al. GC-MS analysis of ether soluble components and determination of amino acids in Indigo naturalis. Fujian J Tradit Chin Med. 2011;42:54-5.

11. Degani L, Riedo C, Chiantore O. Identification of natural indigo in historical textiles by GC-MS. Anal Bioanal Chem. 2015;407:1695-704.

12. Liao W, Fu CM, Wang JS, et al. Determination of heavy metals in indigo naturalis by icp-ms. West China J Pharm Sci. 2010;25:483-4.

13. Zhou W, Zhang XY. Research progress of Chinese herbal medicine Radix isatidis (banlangen). Am J Chin Med. 2013;41:743-64.

14. Han X, Zhang DK, Guo YM, et al. Screening and evaluation of commonlyused anti-influenza Chinese herbal medicines based on anti-neuraminidase activity. Chin J Nat Med. 2016;14:794-800.

15. Zhang T, Huang H-Z, Xu R-C, et al. An anti-influenza virus activitycalibrated chemical standardization approach for quality evaluation of indigo naturalis. Anal Methods, 2019; 11: 1.

16. Shi Y, Wei F, Ma SC. Discussion on source,preparation and quality of Indigo Naturalis. Chin J Chin Mater Med. 2019;44:608-13.

17. Afsheen N, Ur K, Jahan R. N., et al. Cardioprotective and Metabolomic Profiling of Selected Medicinal Plants against Oxidative Stress. Oxid Med Cell Longev. 2018;2018:9819360.

18. Du LY, Zhou LB, Zong SQ, et al. Experimental Study on the Effect of Qingdai Granule on Resisting Lipid Peroxidation. Chin J Integr Tradit West Med on Digest. 2003;11:144-5.

19. Lin YK, Chen HW, Yang SH, et al. Protective effect of indigo naturalis extract against oxidative stress in cultured human keratinocytes. J Ethnopharmacol. 2012;139:893-6.

20. Zhao G, LiT, Qu X, et al. Optimization of ultrasound-assisted extraction of indigo and indirubin from Isatis indigotica Fort. and their antioxidant capacities. Food Sci Biotechnol. 2017;26:1313-23.

21. Luo $C, X u X$, Wei $X$, et al. Natural medicines for the treatment of fatigue: Bioactive components, pharmacology, and mechanisms. Pharmacol Res. 2019;148:104409.
22. Farias-Silva E, Cola M, Calvo TR, et al. Antioxidant activity of indigo and its preventive effect against ethanol-induced DNA damage in rat gastric mucosa. Planta Med. 2007:73:1241-6.

23. Lai JL, Liu YH, Liu C, et al. Indirubin Inhibits LPS-Induced Inflammation via TLR4 Abrogation Mediated by the NF-kB and MAPK Signaling Pathways. Inflammation. 2017;40:1-12.

24. Li R, Jia Z, Trush MA. Defining ROS in Biology and Medicine. React Oxyg Species (Apex). 2016;1:9-21.

25. Qi T, Li H, Li S. Indirubin improves antioxidant and anti-inflammatory functions in lipopolysaccharide-challenged mice. Oncotarget. 2017:8:36658-63.

26. Ozawa K, Mori D, Hatanaka A, et al. Comparison of the anti-colitis activities of Qing Dai/Indigo Naturalis constituents in mice. J Pharmacol Sci. 2020;142:148-56

27. Chen XL, Du LY, Liu QF, et al. Qingdai Granules down-regulate TLR2, TLR4 mRNA expression in the colonic mucosa of rats with ulcerative colitis. Chin J Integr Tradit West Med on Digest. 2011;19:14-7.

28. Kawai S, lijima H, Shinzaki S, et al. Indigo Naturalis ameliorates murine dextran sodium sulfate-induced colitis via aryl hydrocarbon receptor activation. J Gastroenterol. 2017:52:904-19.

29. WQ M. K, Y., X, T., et al. The therapeutic effect and mechanism of indigo on dextran sulfate sodium induced ulcerative colitis in mice. Pro Anat Sci. 2019;25:146-9.

30. Sethi G, Ahn KS, Sandur SK, et al. Indirubin enhances tumor necrosis factor-induced apoptosis through modulation of nuclear factor-kappa B signaling pathway. J Biol Chem. 2006;281:23425-35.

31. Gao W, Zhang L, Wang $X$, et al. The combination of indirubin and isatin attenuates dextran sodium sulfate induced ulcerative colitis in mice. Biochem Cell Biol. 2018:96:636-45.

32. Lai JL, Liu YH, Peng YC, et al. Indirubin Treatment of LipopolysaccharideInduced Mastitis in a Mouse Model and Activity in Mouse Mammary Epithelial Cells. Mediators Inflamm. 2017;2017:3082805.

33. Gao W, Guo Y, Wang C, et al. Indirubin ameliorates dextran sulfate sodium-induced ulcerative colitis in mice through the inhibition of inflammation and the induction of Foxp3-expressing regulatory $T$ cells. Acta Histochem. 2016;118:606-14.

34. Lin YK, Leu YL, Huang TH, et al. Anti-inflammatory effects of the extract of indigo naturalis in human neutrophils. J Ethnopharmacol. 2009:125:51-8.

35. Tsai YC, Lee CL, Yen HR. Antiviral Action of Tryptanthrin Isolated from Strobilanthes cusia Leaf against Human Coronavirus NL63. Biomolecules. 2020;10:366.

36. Jie C, Luo Z, Chen $\mathrm{H}$, et al. Indirubin, a bisindole alkaloid from Isatis indigotica, reduces H1N1 susceptibility in stressed mice by regulating MAVS signaling. Oncotarget. 2017:8:105615-29.

37. Kataoka M, Hirata K, Kunikata T, et al. Antibacterial action of tryptanthrin and kaempferol, isolated from the indigo plant (Polygonum tinctorium Lour.), against Helicobacter pylori-infected Mongolian gerbils. J Gastroenterol. 2001;36:5-9.

38. Chiang YR, Li A, Leu YL, et al. An in vitro study of the antimicrobial effects of indigo naturalis prepared from Strobilanthes formosanus Moore. Molecules. 2013:18:14381-96.

39. Bandekar PP, Roopnarine KA, Parekh VJ, et al. Antimicrobial activity of tryptanthrins in Escherichia coli. J Med Chem. 2010;53:3558-65.

40. Andreazza NL, de Lourenço CC, Stefanello M, et al. Photodynamic antimicrobial effects of bis-indole alkaloid indigo from Indigofera truxillensis Kunth (Leguminosae). Lasers Med Sci. 2015;30:1315-24.

41. Ponnusamy K, Ramasamy M, Savarimuthu I, et al. Indirubin potentiates ciprofloxacin activity in the NorA efflux pump of Staphylococcus aureus. Scand J Infect Dis. 2010;42:500-5.

42. Sravanthi TV, Manju SL. Indoles - A promising scaffold for drug development. Eur J Pharm Sci. 2016;91:1-10

43. Hutchinson EC. Influenza Virus. Trends Microbiol. 2018;26:809-10.

44. Mak NK, Leung CY, Wei XY, et al. Inhibition of RANTES expression by indirubin in influenza virus-infected human bronchial epithelial cells. Biochem Pharmacol. 2004;67:167-74.

45. Lin CW, Tsai FJ, Tsai CH, et al. Anti-SARS coronavirus 3C-like protease effects of Isatis indigotica root and plant-derived phenolic compounds. Antiviral Res. 2005;68:36-42.

46. Lin YK, See LC, Huang YH, et al. Comparison of indirubin concentrations in indigo naturalis ointment for psoriasis treatment: a randomized, doubleblind, dosage-controlled trial. Br J Dermatol. 2018;178:124-31. 
47. Kim MH, Choi YY, Yang G, et al. Indirubin, a purple 3,2- bisindole, inhibited allergic contact dermatitis via regulating Thelper (Th)-mediated immune system in DNCB-induced model. J Ethnopharmacol. 2013;145:214-9.

48. Adachi S, Hoshi N, Inoue J, et al. Indigo Naturalis Ameliorates OxazoloneInduced Dermatitis but Aggravates Colitis by Changing the Composition of Gut Microflora. Int Arch Allergy Immunol. 2017;173:23-33.

49. Xie XJ, Di TT, Wang Y, et al. Indirubin ameliorates imiquimod-induced psoriasis-like skin lesions in mice by inhibiting inflammatory responses mediated by IL-17A-producing $\gamma \delta$ T cells. Mol Immunol. 2018;101:386-95.

50. Zhang A, Qu Y, Zhang B, et al. The different effects of indirubin on effector and CD4 + CD25 + regulatory T cells in mice: potential implication for the treatment of autoimmune diseases. J Mol Med (Berl). 2007;85:1263-70.

51. Man Y, Wang YX, Zhu SY, et al. Indirubin inhibits ATP-induced phagocytosis attenuation, ROS production and cell death of macrophages. Acta Pharm Sin. 2012:47:45-50.

52. Kim SA, Kim YC, Kim SW, et al. Antitumor activity of novel indirubin derivatives in rat tumor model. Clin Cancer Res. 2007;13:253-9.

53. Eisenbrand G, Hippe F, Jakobs S, et al. Molecular mechanisms of indirubin and its derivatives: novel anticancer molecules with their origin in traditional Chinese phytomedicine. J Cancer Res Clin Oncol. 2004;130:627-35.

54. The Cooperation Group of Phase II Clinical Trial of Compound Huangdai Tablet. Phase || clinical trial of compound Huangdai tablets in newly diagnosed acute promyelocytic leukemia. Chin J Hematol, 2006: 801-4.

55. Huang SL, Xiang GA, Wang Y, Lin HX, Fu XBL. Clinical study on the treatment of acute promyelocytic leukemia with Composite Indigo Naturalis tablets. Chin J Hemat. 1995;1:26-8.

56. Wang L, Zhou GB, Liu P, et al. Dissection of mechanisms of Chinese medicinal formula Realgar-Indigo naturalis as an effective treatment for promyelocytic leukemia. Proc Natl Acad Sci U S A. 2008;105:4826-31.

57. Zhang $X$, Song $Y, W u Y$, et al. Indirubin inhibits tumor growth by antitumor angiogenesis via blocking VEGFR2-mediated JAK/STAT3 signaling in endothelial cell. Int J Cancer. 2011;129:2502-11.

58. Chan HL, Yip HY, Mak NK, et al. Modulatory effects and action mechanisms of tryptanthrin on murine myeloid leukemia cells. Cell Mol Immunol. 2009;6:335-42.

59. Shankar GM, AlexW, Nisthul AA, et al. Pre-clinical evidences for the efficacy of tryptanthrin as a potent suppressor of skin cancer. Cell Prolif. 2020;53:e12710.

60. Liao X, Zhou X, Mak NK, et al. Tryptanthrin inhibits angiogenesis by targeting the VEGFR2-mediated ERK1/2 signalling pathway. PLoS One. 2013;8:e82294.

61. Yu ST, Chen TM, Tseng SY, et al. Tryptanthrin inhibits MDR1 and reverses doxorubicin resistance in breast cancer cells. Biochem Biophys Res Commun. 2007:358:79-84

62. Lin YK, Leu YL, Yang SH, et al. Anti-psoriatic effects of indigo naturalis on the proliferation and differentiation of keratinocytes with indirubin as the active component. J Dermatol Sci. 2009;54:168-74.

63. Liu SG, Luo GP, Qu YB, et al. Indirubin inhibits Wnt/ $\beta$-catenin signal pathway via promoter demethylation of WIF-1. BMC Complement Med Ther. 2020;20:250.

64. Xue X, Wu J, Li J, et al. Indirubin attenuates mouse psoriasis-like skin lesion in a CD274-dependent manner: an achievement of RNA sequencing. Biosci Rep. 2018;38:1.

65. Cheng HM, Kuo YZ, Chang CY, et al. The anti-TH17 polarization effect of Indigo naturalis and tryptanthrin by differentially inhibiting cytokine expression. J Ethnopharmacol. 2020;255:112760.

66. Chang HN, Yeh YC, Chueh HY, et al. The anti-angiogenic effect of tryptanthrin is mediated by the inhibition of apelin promoter activity and shortened mRNA half-life in human vascular endothelial cells. Phytomedicine. 2019;58:152879.

67. Chang HN, Huang ST, Yeh YC, et al. Indigo naturalis and its component tryptanthrin exert anti-angiogenic effect by arresting cell cycle and inhibiting Akt and FAK signaling in human vascular endothelial cells. J Ethnopharmacol. 2015;174:474-81.

68. Wang Y, Liu L, Guo Y, et al. Effects of indigo naturalis on colonic mucosal injuries and inflammation in rats with dextran sodium sulphate-induced ulcerative colitis. Exp Ther Med. 2017;14:1327-36.

69. Chen MS, Du LY. Effect of Compound Qingdai Granula on the expression of TGF- $\beta 1$ and VEGF in the colon tissue of rats with ulcerative colitis. Chin J Integr Tradit West Med on Digest. 2013;21:393-6.
70. Wang X, Du LY, Zhang Y, et al. Effects of Compound Qingdai Granules on the expression of MMP-1and TIMP-1 of model rats with ulcerative colitis. Chin J Integr Tradit West Med on Digest. 2013;21:565-8.

71. Liang YN, Yu JG, Zhang DB, et al. Indigo Naturalis Ameliorates Dextran Sulfate Sodium-Induced Colitis in Mice by Modulating the Intestinal Microbiota Community. Molecules. 2019;24:4086.

72. Stahl M, Tallman MS. Differentiation syndrome in acute promyelocytic leukaemia. Br J Haematol. 2019;187:157-62.

73. Gill H, Yim R, Lee HKK, et al. Long-term outcome of relapsed acute promyelocytic leukemia treated with oral arsenic trioxide-based reinduction and maintenance regimens: A 15-year prospective study. Cancer. 2018;124:2316-26.

74. Wang L, Li X, Liu X, et al. Enhancing effects of indirubin on the arsenic disulfide-induced apoptosis of human diffuse large B-cell lymphoma cells. Oncol Lett. 2015;9:1940-6.

75. Xiang Y, Huang SL, Guo AX, et al. The influence on long-term survey of the patients with acute promyelocytic leukemia treated alternatively with compound huangdai tablets and chemotherapy. J Clin Hematol. 2003;1:204-6.

76. Jiao ZZ, Controlled clinical observation of Compound Huangdai Tablet in the intensive therapy of Acute Promyelocytic Leukemia, Guangzhou University of Traditional Chinese Medicine, 2012.

77. Zhu HH, Wu DP, Du X, et al. Oral arsenic plus retinoic acid versus intravenous arsenic plus retinoic acid for non-high-risk acute promyelocytic leukaemia: a non-inferiority, randomised phase 3 trial. Lancet Oncol. 2018;19:871-9.

78. Yang MH, Wan WQ, Luo JS, et al. Multicenter randomized trial of arsenic trioxide and Realgar-Indigo naturalis formula in pediatric patients with acute promyelocytic leukemia: Interim results of the SCCLG-APL clinical study. Am J Hematol. 2018;93:1467-73.

79. Yanovsky RL, Chen H, Leslie S, et al. The Interaction of LILRB2 with HLA-B Is Associated with Psoriasis Susceptibility. J Invest Dermatol. 2020;140:1292-5.e3.

80. Yuan ZZ, Wan X, et al. Observation on the therapeutic effect of Indigo Naturalis on 46 cases of psoriasis. J Tradit Chin Med. 1982;1:43.

81. Lin YK, Chang CJ, Chang YC, et al. Clinical assessment of patients with recalcitrant psoriasis in a randomized, observer-blind, vehicle-controlled trial using indigo naturalis. Arch Dermatol. 2008;144:1457-64.

82. Lin YK, See LC, Chang YC, et al. Treatment of psoriatic nails with indigo naturalis oil extract: a non-controlled pilot study. Dermatology. 2011;223:239-43.

83. Cheng HM, Wu YC, Wang Q, et al. Clinical efficacy and IL-17 targeting mechanism of Indigo naturalis as a topical agent in moderate psoriasis. BMC Complement Altern Med. 2017;17:439.

84. Lin YK, Yen HR, Wong WR, et al. Successful treatment of pediatric psoriasis with Indigo naturalis composite ointment. Pediatr Dermatol. 2006;23:507-10.

85. Liang CY, Lin TY, Lin YK. Successful treatment of pediatric nail psoriasis with periodic pustular eruption using topical indigo naturalis oil extract. Pediatr Dermatol. 2013;30:117-9.

86. Ordás I, Eckmann L, Talamini M, et al. Ulcerative colitis. The Lancet. 2012;380:1606-19.

87. Naganuma M, Sugimoto S, Suzuki H, et al. Adverse events in patients with ulcerative colitis treated with indigo naturalis: a Japanese nationwide survey. J Gastroenterol. 2019;54:891-6.

88. Urushikubo J, Yanai S, Nakamura S, et al. Efficacy of Indigo Naturalis Therapy for Ulcerative Colitis: A Case Series. Intern Med. 2019;58:2299-304.

89. Yoshimatsu Y, Naganuma M, Sugimoto S, et al. Development of an Indigo Naturalis Suppository for Topical Induction Therapy in Patients with Ulcerative Colitis. Digestion. 2020;101:492-8.

90. Naganuma M, Sugimoto S, Mitsuyama K, et al. Efficacy of Indigo Naturalis in a Multicenter Randomized Controlled Trial of Patients With Ulcerative Colitis. Gastroenterology. 2018;154:935-47.

91. Matsuno Y, Hirano A, Torisu T, et al. Short-term and long-term outcomes of indigo naturalis treatment for inflammatory bowel disease. J Gastroenterol Hepatol. 2020;35:412-7.

92. Aten J, Roos A, Claessen N, et al. Strong and selective glomerular localization of CD134 ligand and TNF receptor-1 in proliferative lupus nephritis. J Am Soc Nephrol. 2000;11:1426-38.

93. Chen DY, Chen YM, Wen MC, et al. The potential role of Th17 cells and Th17-related cytokines in the pathogenesis of lupus nephritis. Lupus. 2012;21:1385-96.

\section{Publisher's Note}

Springer Nature remains neutral with regard to jurisdictional claims in published maps and institutional affiliations. 